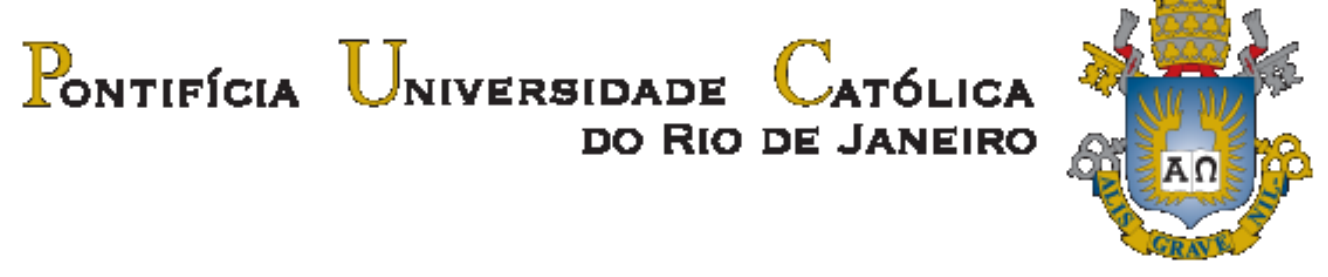

Eduardo G. de O. Vianna

\title{
Estudo numérico do efeito de estenose coronariana no fluxo sanguíneo.
}

\section{Projeto de Graduação}

Projeto final apresentado ao Programa de graduação em Engenharia Mecânica da PUC-Rio como requisito parcial para obtenção do título de Bacharel em Engenharia Mecânica.

Orientador: Profa. Angela Ourivio Nieckele Co-orientador: Dr. Bruno Alvares de Azevedo Gomes

Rio de Janeiro Julho de 2017 


\section{Agradecimentos}

Gostaria de agradecer todos que tornaram essa jornada possível e que me ajudaram a vencer esta trajetória da minha vida.

Agradeço a minha família por sempre me darem apoio incondicional.

Minha namorada Michelle Gottlieb Marra por sempre me alegrar nos momentos mais difíceis e sempre estar ao meu lado.

Agradeço todos os professores por todos os ensinamentos ao longo da minha graduação

À minha orientadora Angela Nieckele Ourives, e co-orientador Bruno Alvares de Azevedo Gomes pela orientação durante a pesquisa e pelos ensinamentos dados necessários para a conclusão deste trabalho.

À todas as amizades que criei durante meus períodos na PUC-Rio.

À toda equipe do departamento de engenharia mecânica. 


\section{Resumo}

Vianna, Eduardo, G.O.; Estudo numérico do efeito de estenose coronariana no fluxo sanguíneo. Rio de Janeiro, 2017. 37p. Projeto Final de Graduação - Departamento de Engenharia Mecânica, Pontifícia Universidade Católica do Rio de Janeiro.

A estenose coronariana provoca alterações no fluxo sanguíneo que participa do processo patológico da doença aterosclerótica. A utilização da dinâmica dos fluidos computacional tem se tornado uma forte ferramenta para auxiliar no diagnóstico de aterosclerose coronariana, evitando que exames mais intrusivos sejam realizados nos pacientes. No presente trabalho determinou-se numericamente o campo de velocidade e pressão em um trecho de uma coronária, utilizando o software comercial Fluent. Investigou-se a possível alteração no padrão do escoamento devido a diversos níveis de lesões nas artérias coronárias. Determinou-se a tensão cisalhante na parede endotelial da artéria coronariana e da queda de pressão entre antes e após a obstrução. Bons resultados foram obtidos ao comparar com dados experimentais. A partir dos resultados do padrão de queda de pressão em relação ao nível de estenose, foi possível a criação de uma equação de quarta ordem para o cálculo do FFR (Fractional Flow Reserve) a partir da redução de área causada pela estenose coronariana.

\section{Palavras chaves:}

Aterosclerose. Artéria coronária. Estenose coronariana. Simulação numérica. 


\section{Abstract}

Vianna, Eduardo, G.O.; Numerical study of the effect of coronary stenosis on blood flow. Rio de Janeiro, 2017. 37p. Final Project - Department of Mechanical Engineering, Pontifícia Universidade Católica do Rio de Janeiro.

Coronary stenosis causes alterations in blood flow that influences the pathological process of atherosclerotic disease. The use of computational fluid dynamics has become a strong tool in aiding the diagnosis of coronary atherosclerosis, avoiding more intrusive procedures in patients. In the present work, the velocity and pressure field were determined numerically, using commercial software Fluent, in a section of a coronary. It was investigated possible change in flow pattern, due to several levels of lesions in the coronary arteries. The shear stress was determined on the endothelial wall of the coronary artery and the pressure drop between before and after the obstruction. Satisfactory results were obtained when comparing with experimental data. From the results of the pressure drop pattern in relation to the stenosis level, it was possible to create a fourth order equation for the calculation of FFR (Fractional Flow Reserve) from the area reduction caused by coronary stenosis.

\section{Key-words:}

Atherosclerosis. Coronary artery. Coronary stenosis. Numerical simulation. 


\section{Sumário}

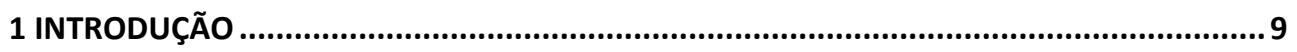

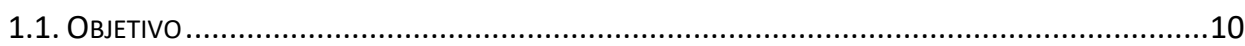

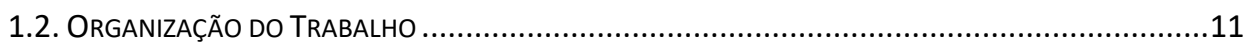

2 REVISÃO BIBLIOGRÁFICA ..................................................................................... 13

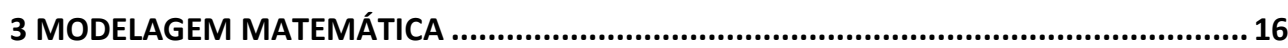

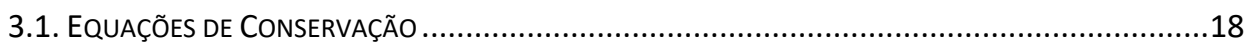

4 MODELAGEM NUMÉRICA ................................................................................. 21

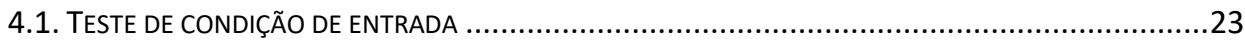

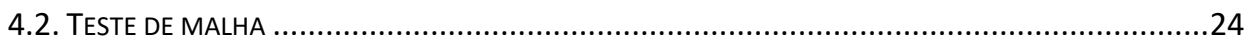

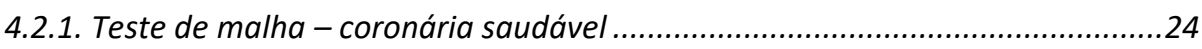

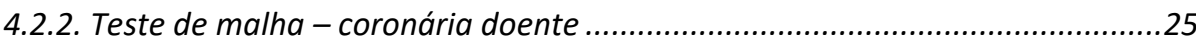

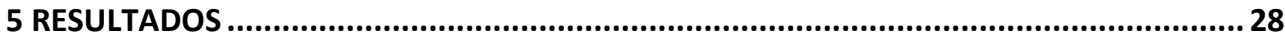

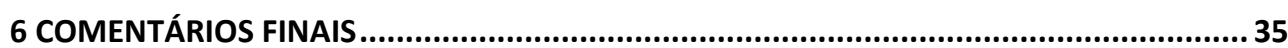

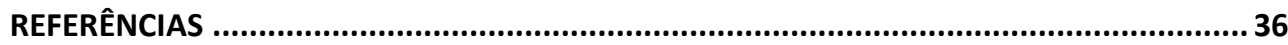




\section{Lista de figuras}

Figura 1.1 -- Cateter de medição na coronária. http://br.sjm.com /storage/ /images/produtos/cardiologia-e-acesso-

vascular_a/products_pressurewirecertus_1.jpg .............................. 9

Figura 1.2 - Representação da estenose coronariana saudável e com

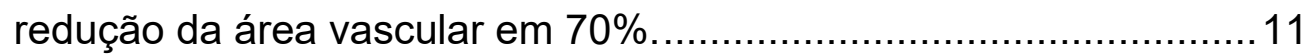

Figura 3.1 - Coronária inteira ................................................... 16

Figura 3.2 - Coronárias em ordem crescente de estenose ...................17

Figura 3.3 - Entrada da coronária do modelo 3D ................................20

Figura 4.1- Perfil da velocidade na entrada ....................................22

Figura 4.2 - Planos para cálculo das pressões...................................22

Figura 4.3 - Influência do perfil de velocidade na entrada da distribuição de pressão ................................................................... 23

Figura 4.4 - Malha grosseira da coronária saudável ...........................24

Figura 4.5 - Gráfico da queda de pressão ......................................25

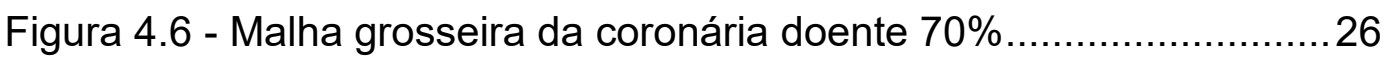

Figura 4.7 -Gráfico da queda de pressão ........................................ 27

Figura 5.1 - Planos de velocidade no plano $+X$, para a coronária saudável e com as diferentes obstruções ............................................29

Figura 5.2 - Tensão cisalhante nas coronárias $(0 \%, 16 \%, 34 \%) \ldots \ldots \ldots \ldots . . . .31$

Figura 5.3 - Tensão cisalhante na coronária $(50 \%, 60 \%, 70 \%)$............... 31

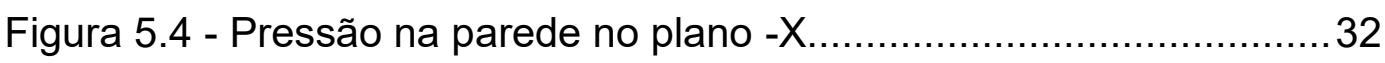

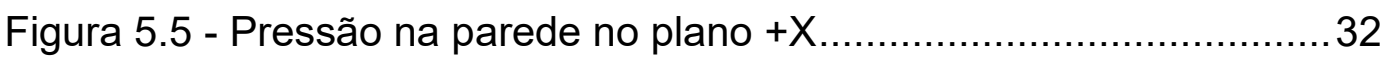

Figura 5.6 - A relação entre $P d / P a$ e a direção axial $S$. .......................33

Figura 5.7 - Gráfico do perfil de queda de pressão ...............................34 


\section{Lista de tabelas}

Tabela 4.1 - Número de nós das malhas. Teste para coronária saudável24

Tabela 4.2 -Tabela de número de nós das malhas ...............................26 


\section{Nomenclatura}

$\begin{array}{ll}A_{t} & \text { Área transversal } \\ C F D & \text { Dinâmica dos fluidos computacionais } \\ u & \text { Componente da velocidade em x } \\ v & \text { Componente da velocidade em y } \\ w & \text { Componente da velocidade em z } \\ \rho & \text { Densidade do fluido } \\ D_{h} & \text { Diâmetro hidráulico } \\ F F R & \text { Fluxo de reserva } \\ P_{m} & \text { Perímetro molhado } \\ p & \text { Pressão } \\ p_{a} & \text { Pressão aórtica } \\ p_{d} & \text { Pressão distal } \\ r_{a} & \text { Raio na direção x } \\ r_{b} & \text { Raio na direção y } \\ \alpha & \text { Razão de obstrução } \\ u_{i} & \text { Velocidade com índice } i \\ u_{m} & \text { Velocidade média } \\ \mu & \text { Viscosidade }\end{array}$




\section{Introdução}

A aterosclerose é uma doença inflamatória, com formação de placas de gordura, cálcio e outros elementos na parede das artérias do coração e outras localidades do corpo humano. Ela é caracterizada pelo estreitamento e enrijecimento das artérias devido ao acúmulo de gordura em suas paredes.

Um dos métodos mais tradicionais para o diagnóstico da aterosclerose coronariana é feito por técnicas invasivas de coronariografia. Este procedimento exige a introdução de um cateter pela rede arterial e a infusão de contraste iodado na rede arterial das artérias coronárias. Em lesões ateroscleróticas duvidosas, uma técnica de medição da queda de pressão é realizada, denominada de Reserva de Fluxo (FFR - Fractional Flow Reserve, (James K. Min, et al., 2012) e ilustrado na Figura 1.1. Variáveis com importância hemodinâmica nas lesões ateroscleróticas são a velocidade de fluxo, pressão e estresse de cisalhamento na parede vascular.

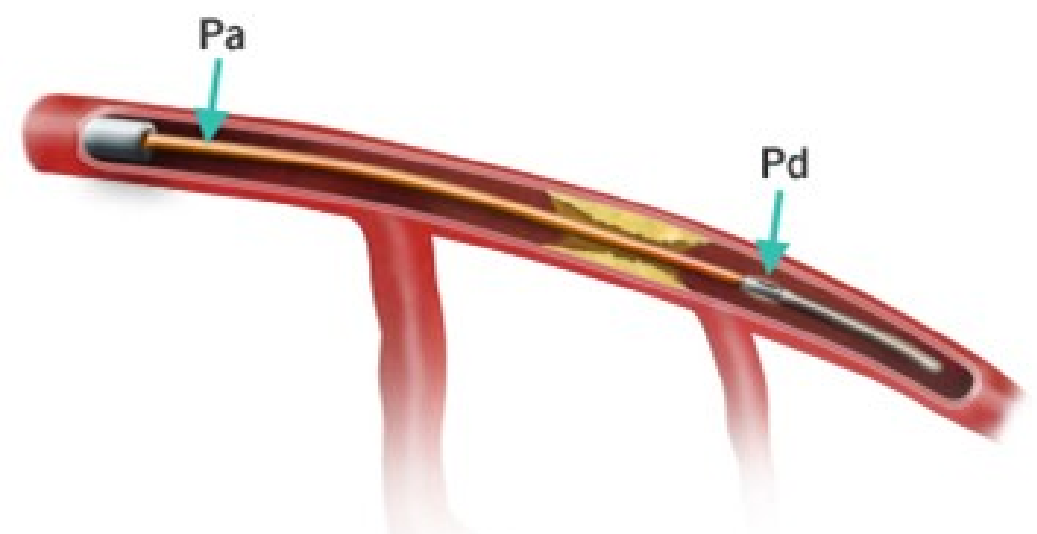

Figura 1.1 -- Cateter de medição na coronária. http://br.sjm.com /storage/ /images/produtos/cardiologia-e-acesso-vascular_a/products_pressurewirecertus_1.jpg 
A utilização da dinâmica dos fluidos computacional tem se tornado uma forte ferramenta para auxiliar no diagnóstico de aterosclerose coronariana, evitando que procedimentos mais intrusivos sejam realizados nos pacientes.

\subsection{Objetivo}

A proposta deste trabalho é investigar a influência da estenose coronariana na hemodinâmica, principalmente focando-se no FFR, principal ferramenta para a determinação da gravidade de lesões coronarianas. A FFR é uma técnica muito utilizada para determinar o comprometimento hemodinâmico das lesões ateroscleróticas. O cálculo é feito dividindo-se a pressão distal à lesão coronária $(\mathrm{Pd})$ pela pressão aórtica $(\mathrm{Pa})$.

A geometria da coronária foi obtida a partir de resultados de exame de imagem realizado pelo paciente. Visando avaliar a diferença no escoamento de diferentes percentuais de obstrução, criou-se numericamente coronárias com diferentes graus de obstrução. A Figura 1.2, apresenta uma imagem da coronária saudável (a) e uma com lesão estenótica de $70 \%$ (b).

Para a modelagem foi utilizado tomografia computadorizada, uma tecnologia que permite a produção de uma sequência de imagens, com espessuras milimétricas, transversais dos órgãos, utilizadas para a geração do modelo vascular 3D. Posteriormente, um protocolo de segmentação de imagens foi implementado com a utilização do software Fiji (ImageJ). A partir deste arquivo é possível a manipulação do modelo 3D e a geração da malha computacional através do software Fluent da ANSYS, EUA. Este processo possibilita a realização do cálculo dos parâmetros desejados para a análise do problema em questão.

O modelo vascular 3D foi simplificado, selecionando-se um segmento da árvore coronariana com o intuito de diminuir o tempo computacional e otimizar a convergência do modelo. O modelo coronariano possuía aproximadamente $16 \mathrm{~mm}$ de comprimento. O estreitamento do lúmen 
vascular foi representado por uma diminuição no diâmetro da coronária na geometria 3D, medidos em 16\%, 34\%, 50\%, 60\% e 70\% de redução da área original da seção transversal.

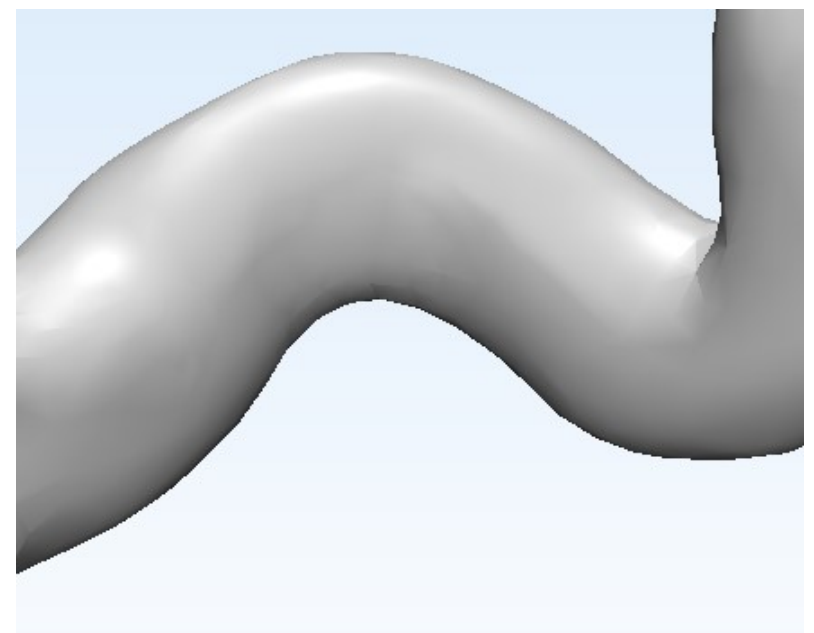

(a) coronária saudável

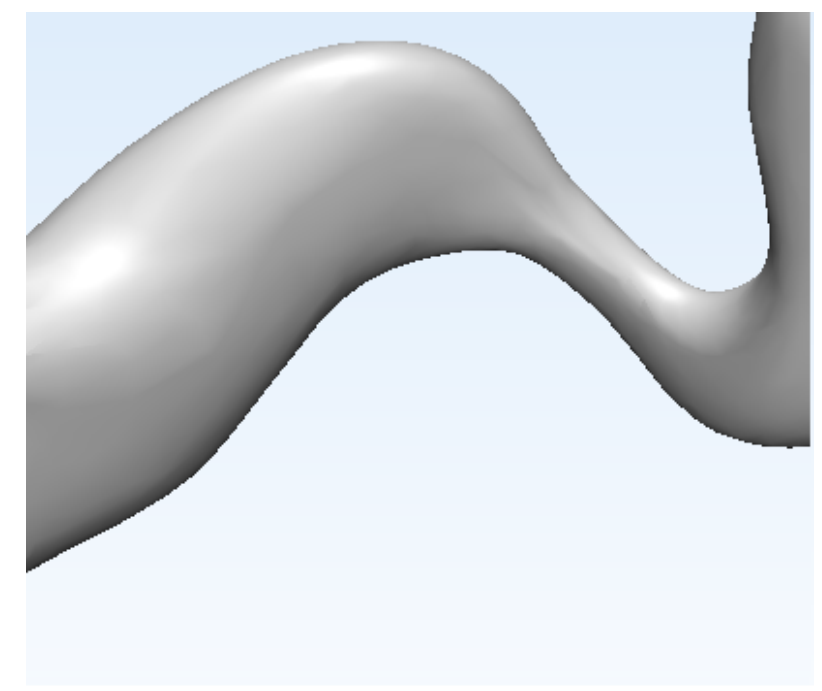

(b) coronária doente com obstrução.

Figura 1.2 - Representação da estenose coronariana saudável e com redução da área vascular em $70 \%$.

\subsection{Organização do Trabalho}

O presente texto foi dividido em seis capítulos. No próximo capítulo será apresentada uma revisão bibliográfica dos vários estudos sobre a aterosclerose coronariana. No Capítulo 3 apresenta-se as equações governantes e os modelos constitutivos do problema estudado. No 
Capítulo 4 é feita a modelagem numérica e em seguida foi feito dois testes de malha para a validação de malhas. O Capítulo 5 apresenta os resultados obtidos de campo de velocidade, tensão cisalhante e pressão. No Capítulo 6 são apresentadas as conclusões e sugestões. 


\section{2 \\ Revisão Bibliográfica}

A técnica FFR (FFR - Fractional Flow Reserve) tem sido muito empregada para diagnóstico de lesões sérias na coronária. Porém, é uma técnica invasiva, com risco e causando desconforto ao paciente. Um dos problemas encontrados na utilização da técnica FFR surge quando várias estenoses estão presentes na coronária. (Nico H.J. Pijls, et al., 2000) faz um estudo com 32 pacientes e cada um com estenoses em série em uma artéria coronária. Foi desenvolvida uma fórmula para prever o resultado, além de compará-lo com a medição experimental. Foi demonstrado que sem as correções implementadas, as estenoses seriam significativamente superestimadas.

Em 2007, Feijó realizou um estudo numérico de um modelo tridimensional do escoamento sanguíneo no interior da aorta abdominal. Os campos de pressão, velocidade e o campo de deslocamento da artéria foram obtidos utilizando empregando o software FLUENT ANSYS. Utilizou-se dados experimentais da artéria aorta abdominal para validação do modelo numérico. Bons resultados foram obtidos quando comparado com dados experimentais.

(Kern, et al., 2010) buscaram integrar conceitos de fisiologia integrada da coronária para a angiografia. A racionalização vem de dois pontos principais, a revascularização da coronária depende fortemente da significância hemodinâmica da lesão e a angiografia coronária frequentemente falha em identificar precisamente a hemodinâmica das estenoses coronarianas, principalmente as que apresentam estenoses entre $30 \%$ e $80 \%$.

Em (Hajati, et al., 2012) são avaliados os parâmetros hemodinâmicos nas coronárias utilizando-se de CFD 3D, com uma simulação paciente-específica gerada através de uma tomografia 
computadorizada. O fluxo de massa, tensão cisalhante na parede, gradiente espacial do cisalhamento e pressão na parede, foram os parâmetros quantificados para a análise hemodinâmica. Foi demonstrado que CFD é um método válido de determinar a hemodinâmica de um paciente de forma não invasiva.

O objetivo principal de (James K. Min, et al., 2012) é verificar a acurácia do FFRct, em diagnósticos de estenoses coronarianas hemodinamicamente significativas. Aplicou-se um estudo em 252 pacientes com suspeita ou doença coronariana estabelecida. $O$ estudo não atinge o seu objetivo primário de determinação de diagnóstico com precisão, porém foi concluído que a junção de FFRct e CT (angiotomografia coronariana) exibem resultados mais precisos quando comparado a exames feitos com apenas CT.

(Charles A. Taylor, et al., 2013) se aprofunda no assunto de FFRстA, que é a reserva de fluxo derivado da angiotomografia coronariana, utilizando-se de CFD para a assistência dos resultados. Os autores concluíram que apesar do FFRCTA oferecer ótimos resultados, existem limitações, pois pode ser muito dependente da qualidade do modelo anatômico, e este pode ser influenciado por vários artefatos, incluindo, calcificação, movimento e registro inadequado da imagem.

O objetivo do trabalho de (NXT Trial, 2014), assim como o estudo anterior, foi determinar o desempenho de diagnóstico, mas desta vez comparando com o método invasivo, no qual um cateter é utilizado para a medição direta da pressão arterial. Os autores concluíram que o FFR computadorizado apresentou um diagnóstico bem preciso quando comparado ao diagnóstico anatômico. Além disso, o FFR computadorizado gerou um aumento na especificidade dos resultados.

(Nørgaard, et al., 2015) avaliaram o desempenho de diagnóstico de FFR computadorizado (FFRctA), não invasivo, em comparação a angiotomografia computadorizada. Para quantificar a calcificação pela angiotomografia utilizaram-se do método de Agatston score, método criado por Arthur Agatston para a medição de cálcio nas artérias 
coronárias. Este foi utilizado em 214 pacientes suspeitos de ter doença coronária. Foi concluído que o FFR computadorizado apresentou uma melhoria significativa no diagnóstico quando comparado a angiotomografia computadorizada em pacientes com altos Agatston score.

Em estudos recentes a angiotomografia coronariana computadorizada detectou um contraste maior em áreas com lesão estenótica, porém, o mecanismo físico para esses gradientes não é claro. (Eslami, et al., 2015) utilizam CFD para investigar a causa. A hipótese de que um fluxo de maior velocidade diminui a concentração de contraste. Uma velocidade de escoamento mais baixa aumentaria a concentração de contraste, sendo confirmada pelo modelo numérico. A explicação sobre a maior quantidade de contraste em áreas com lesão, é devido a uma resistência hidráulica aumentada na região da estenose.

Como podemos observar na revisão bibliográfica, a utilização de CFD e conhecimento em mecânica dos fluidos em problemas médicos pode assistir na prevenção e diagnóstico de doenças, e inclusive aprimorar seu entendimento. Neste caso em particular, a área de estenose em coronárias um aprofundamento maior é necessário, o que é uma grande motivação para o presente estudo. 
3

\section{Modelagem Matemática}

O primeiro passo para ser possível determinar o escoamento através da coronária, consiste na definição da geometria de interesse. Para a criação da geometria, utilizou-se o software aberto Fiji (ImageJ) para converter as imagens geradas pela tomografia computadorizada em uma geometria 3D da coronária de um paciente. Na Figura 3.1 podemos ver a geometria da coronária inteira, uma seção representativa desta foi selecionada e removida a partir da ferramenta de corte do Materialise 3Matic, e então, cada nível de estenose foi gerado individualmente no mesmo software

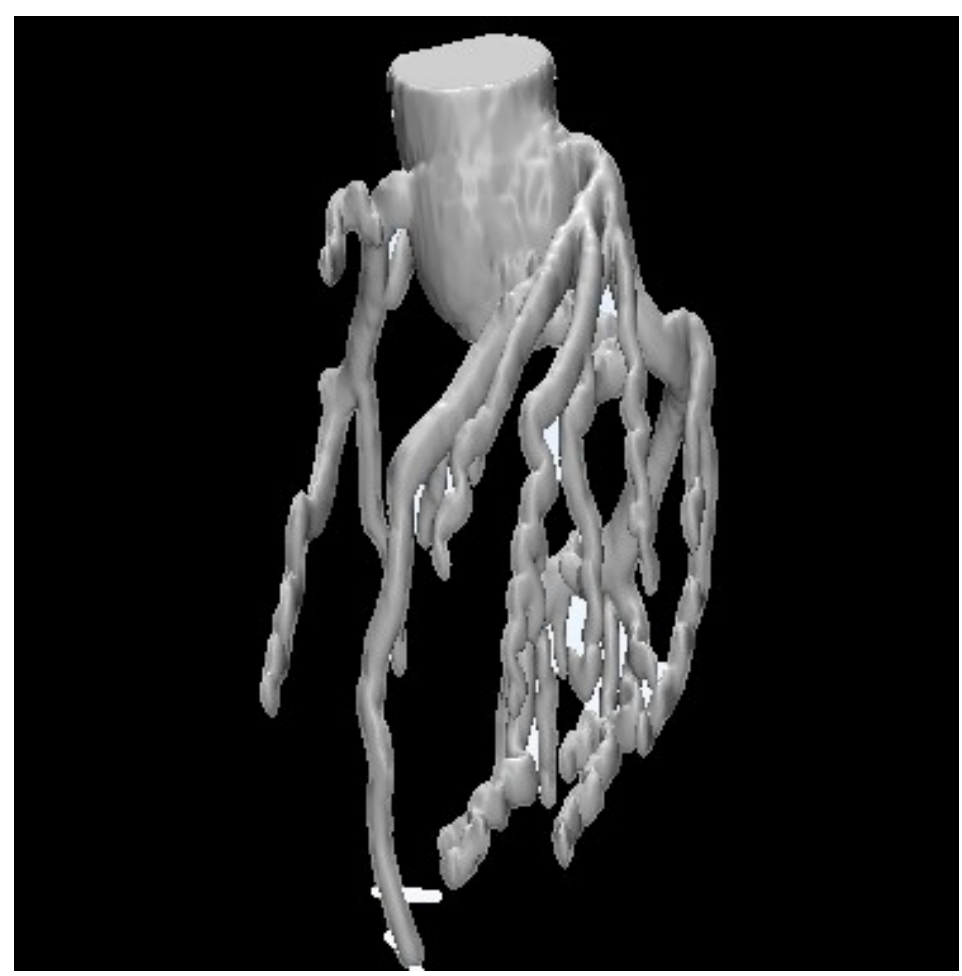

Figura 3.1 - Coronária inteira

As doenças estão representadas na Figura 3.2, em ordem crescente de redução de área, isto é, 0\%, 16\%, 34\%, 50\%, 60\% e 70\%. A estenose está localizada em aproximadamente $4 \mathrm{~mm}$ da entrada. Nestas figuras, 0 
fluxo sanguíneo vai da esquerda para a direita como indicado por uma seta na primeira imagem.

Após a criação das geometrias no 3-matic, com o Design Modeler da ANSYS faz-se alguns ajustes como definir as coordenadas de entrada como o ponto $(0,0,0)$ no centroide do plano da entrada e simplificar as faces de entrada e saída.

Em seguida o software Mesh do ANSYS foi utilizado para a criação das malhas para cada geometria, utilizadas para a realização das simulações no Fluent.

$\mathrm{Na}$ próxima seção as equações de conservação que regem o problema são apresentadas, juntamente com as condições de contorno.

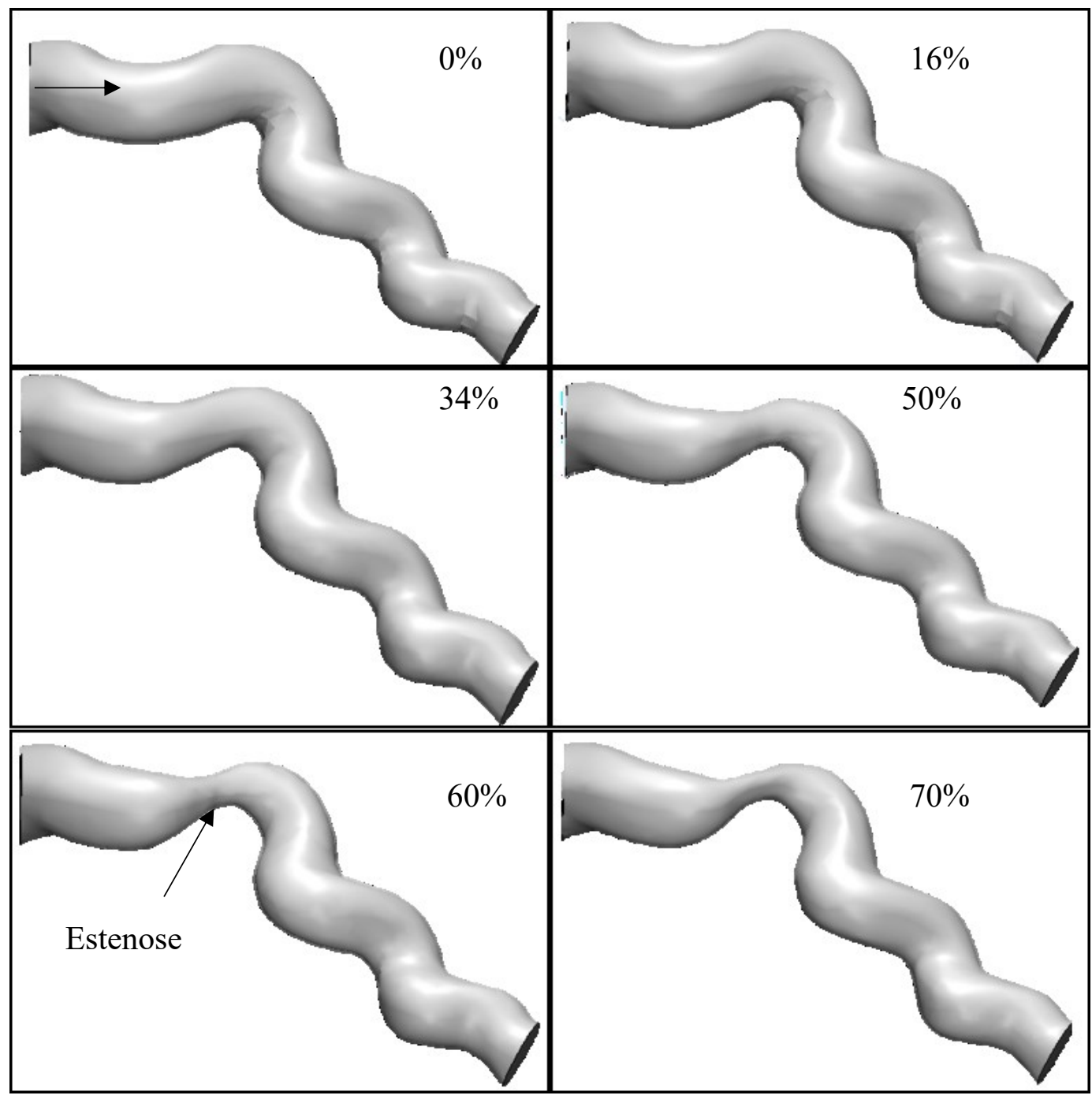

Figura 3.2 - Coronárias em ordem crescente de estenose 


\subsection{Equações de Conservação}

Para determinar o escoamento, diversas hipóteses foram definidas, as quais são descritas a seguir.

- A força gravitacional foi desprezada, pois as forças de pressão são prevalentes.

- O sangue é considerado um fluido incompressível por não apresentar variações de densidade nas condições de operação (Feijó, 2007).

- Apesar de termos um escoamento pulsátil e transiente ao consideramos o fluxo na artéria coronária, o escoamento foi considerado como permanente. Esta hipótese é válida pois, o resultado principal é o cálculo do FFR, o qual é realizado a partir da hiperemia reativa no leito vascular (James K. Min, et al., 2012).

- As paredes da coronária foram consideradas não complacentes, esta hipótese é razoável para um fluxo permanente.

- Considerou-se ainda que o sangue é um fluido Newtoniano, com viscosidade molecular constante (Feijó, 2007)

- Finalmente, devido as pequenas dimensões da coronário, considerouse que o escoamento ocorre no regime laminar.

Este escoamento é governado pela geometria, isto é, percentual de obstrução e número de Reynolds definido como:

$$
R e=\frac{\rho w_{m} D_{h}}{\mu}
$$

onde $w_{m}$ é a velocidade média na entrada, $\rho$ é a massa específica e $\mu$ a viscosidade molecular e $D_{h}$ é o diâmetro hidráulico, definido como

$$
D_{h}=\frac{4 A_{t}}{P_{m}}
$$

sendo $A_{t}$ a área transversal da seção de entrada da coronária e $P_{m}$ é o perímetro molhado. 
Com as hipóteses apresentadas a equação de conservação de massa (Fox, et al., 2011) em coordenadas cartesianas pode ser escrita como

$$
\frac{\partial u}{\partial x}+\frac{\partial v}{\partial y}+\frac{\partial w}{\partial z}=0
$$

onde $u, v$ e $w$ são os componentes de velocidade nas direções $\mathrm{x}, \mathrm{y}$ e $\mathrm{z}$, respectivamente.

Os três componentes da equação de quantidade de movimento linear para um fluido Newtoniano (equação de Navier-Stokes) para fluidos incompressíveis, com viscosidade constante, podem ser escritos em coordenadas cartesianas como

$$
\begin{aligned}
& \rho\left(u \frac{\partial u}{\partial x}+v \frac{\partial u}{\partial y}+w \frac{\partial u}{\partial z}\right)=-\frac{\partial p}{\partial x}+\mu\left(\frac{\partial^{2} u}{\partial x^{2}}+\frac{\partial^{2} u}{\partial y^{2}}+\frac{\partial^{2} u}{\partial z^{2}}\right) \\
& \rho\left(u \frac{\partial v}{\partial x}+v \frac{\partial v}{\partial y}+w \frac{\partial v}{\partial z}\right)=-\frac{\partial p}{\partial y}+\mu\left(\frac{\partial^{2} v}{\partial x^{2}}+\frac{\partial^{2} v}{\partial y^{2}}+\frac{\partial^{2} v}{\partial z^{2}}\right) \\
& \rho\left(u \frac{\partial w}{\partial x}+v \frac{\partial w}{\partial y}+w \frac{\partial w}{\partial z}\right)=-\frac{\partial p}{\partial z}+\mu\left(\frac{\partial^{2} w}{\partial x^{2}}+\frac{\partial^{2} w}{\partial y^{2}}+\frac{\partial^{2} w}{\partial z^{2}}\right)
\end{aligned}
$$

Para resolver essas equações é preciso definir as condições de contorno, o que é apresentado a seguir.

Considerou-se que a velocidade é conhecida na entrada da coronária. Nas paredes, foi imposta a condição de não deslizamento e na saída desprezou-se o fluxo difusivo. A pressão foi definida na entrada como igual a pressão do coração.

Para prescrever a velocidade na entrada, considerou-se duas possibilidades. Na primeira, um perfil uniforme de velocidade foi prescrito. No entanto, como estamos selecionando um segmento da coronária é mais razoável assumir que escoamento se encontra hidrodinamicamente desenvolvido na entrada do domínio computacional. Portanto, assumimos um perfil de velocidade parabólica para a entrada da coronária. 
A entrada, como visto na Figura 3.3, é muito irregular, portanto aproximamos a entrada por uma elipse para simplificar o perfil de velocidade.

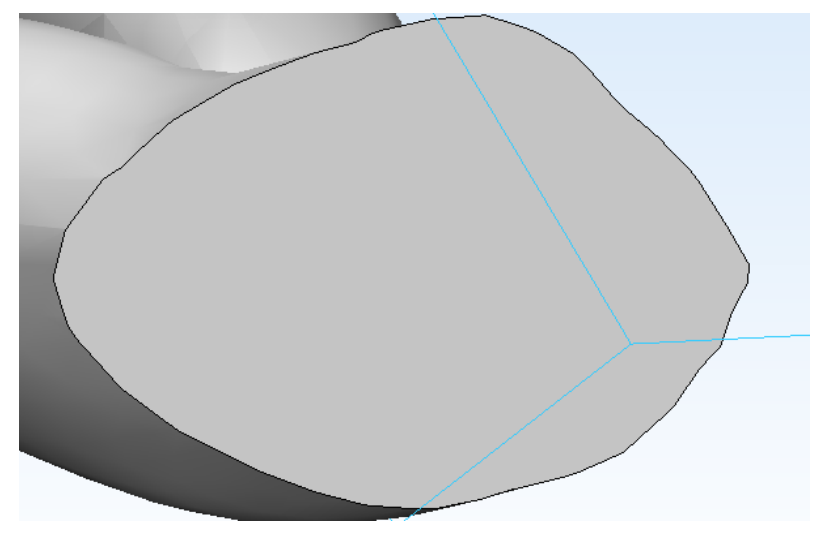

Figura 3.3 - Entrada da coronária do modelo 3D

De acordo com (Rohsenow, et al., 1998) o perfil de velocidade para uma elipse é dado por:

$$
w=2 w_{m}\left[1-\frac{x^{2}}{r_{a}^{2}}+\frac{y^{2}}{r_{b}^{2}}\right]
$$

onde $r_{a}$ e $r_{b}$ são os eixos principais da elipse.

Considerando a seção de entrada da coronária como uma elipse, tem-se que a área da seção transversal é $A_{t}=\pi \mathrm{r}_{\mathrm{a}} \mathrm{r}_{\mathrm{b}}$. O perímetro molhado da elipse é difícil de ser calculado exatamente, podendo ser aproximado pro

$$
P_{m}=\pi\left(r_{a}+r_{b}\right)\left[1+\frac{3 h}{10+\sqrt{4-3 h}}\right] \quad ; h=\frac{\left(r_{a}-r_{b}\right)^{2}}{\left(r_{a}+r_{b}\right)^{2}}
$$

Resultando na seguinte expressão para o diâmetro hidráulico

$$
D_{h}=\frac{4 r_{a} r_{b}}{\left(r_{a}+r_{b}\right)}\left[1+\frac{3 h}{10+\sqrt{4-3 h}}\right]^{-1}
$$




\section{4 \\ Modelagem Numérica}

Para a resolução das equações apresentadas no capítulo anterior, foi selecionado o software Fluent 18 da ANSYS.

O método numérico utilizado pelo software é o de volumes finitos, (Patankar, 1980) este método consiste em dividir o domínio computacional em volumes de controles e em seguida integrar as equações governantes para cada volume.

A avaliação do fluxo difusivo e convectivo nas faces do volume foi realizada utilizando o esquema "Power Law" (Patankar, 1980). Para o esquema de pressão um método de $2^{\mathrm{a}}$ ordem foi utilizado. $\mathrm{O}$ algoritmo SIMPLE foi utilizado para a resolução do acoplamento pressãovelocidade.

O sistema algébrico resultante foi resolvido com GS linha-linha e o algoritmo Multigrid para acelerar a convergência

A convergência foi considerada quando todos os resíduos das equações de conservação, continuidade e quantidade de movimento linear nas direções $x, y$ e $z$, tiveram seus valores absolutos inferiores a $10^{-6}$

Para a definição da condição de contorno parabólica, correspondente a um perfil de velocidade hidrodinamicamente desenvolvido. Foi necessário utilizar uma User Defined Function (UDF) no Fluent, para definir o perfil de velocidade. Esta função foi escrita em C e compilada para ser incorporada ao software. A Figura 4.1 ilustra o perfil de velocidade parabólico prescrito na entrada.

Testes de malha foram realizados, para a definição da malha adequada a ser utilizada nas simulações, visando selecionar uma malha tal que a solução independa do número de volumes de controle. Dois 
testes foram realizados, o primeiro para uma coronária saudável e o segundo para a coronária com obstrução.
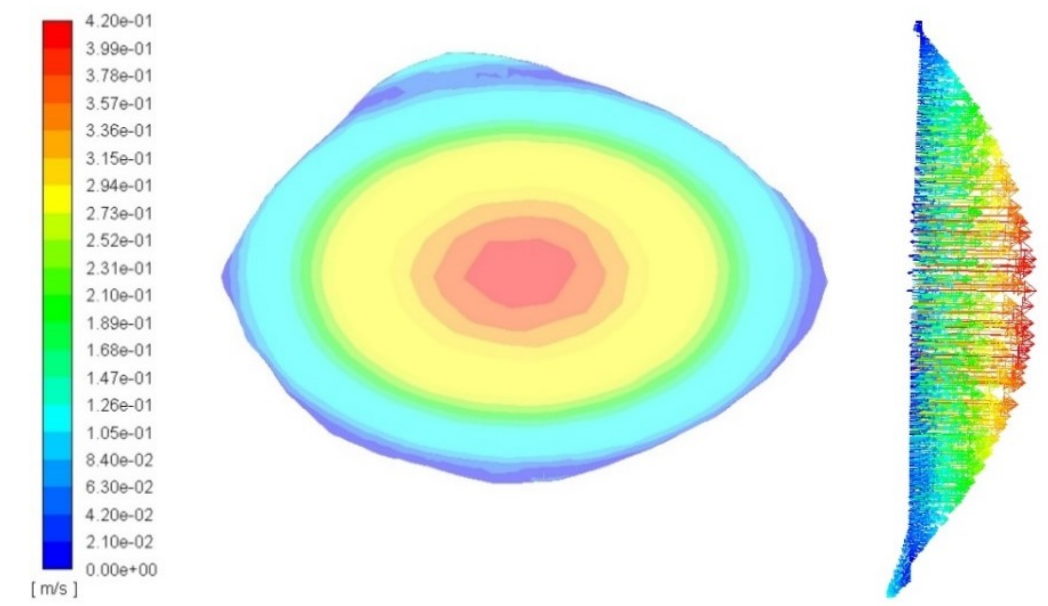

Figura 4.1- Perfil da velocidade na entrada

Na Figura 4.2 é apresentado os planos utilizados para os cálculos das pressões representados por linhas curvas na parede coronariana. Estes planos foram criados a partir da ferramenta de criação de planos do Fluent. Cada ponto apresentado nos gráficos a seguir de FFR, como os da Figura 4.3, representam a média das pressões medidas no plano. Vale lembrar que o FFR referente a estenose é calculado a partir de uma pressão distal a mesma e pressão aórtica.

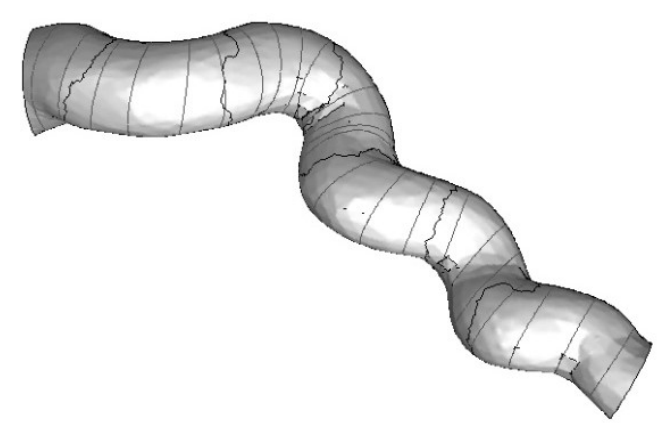

Figura 4.2 - Planos para cálculo das pressões

Algumas observações importantes é que o segmento apresenta muitas curvas, portanto a visualização não é totalmente clara e a coronária também apresenta algumas linhas mais escuras, estas são apenas marcações na malha feito pelo próprio Fluent. 


\subsection{Teste de condição de entrada}

Visando avaliar a influência do perfil de velocidade na entrada da seção de teste da coronária, avaliou-se a distribuição de pressão ao longo da coronária, isto é, ao longo dos planos criados ilustrados na Figura 4.2.

A Figura 4.3 apresenta a comparação entre os perfis de pressão ao longo da coronária, considerando os perfis uniforme e parabólico. Investigou-se uma coronária saudável e doente. Observa-se uma queda de pressão menor na região de entrada para o caso de escoamento parabólico. Este resultado é esperado, devido ao desenvolvimento do escoamento quando o perfil uniforme é utilizado.

Analisando a Figura 4.3 observa-se que a queda de pressão devido a obstrução (parâmetro fundamental para o teste FFR) sobre influência muito pequena do perfil de velocidade na entrada. Porém, como o perfil de velocidade parabólico é mais realista, este foi selecionado para ser utilizado nas análises realizadas neste trabalho.

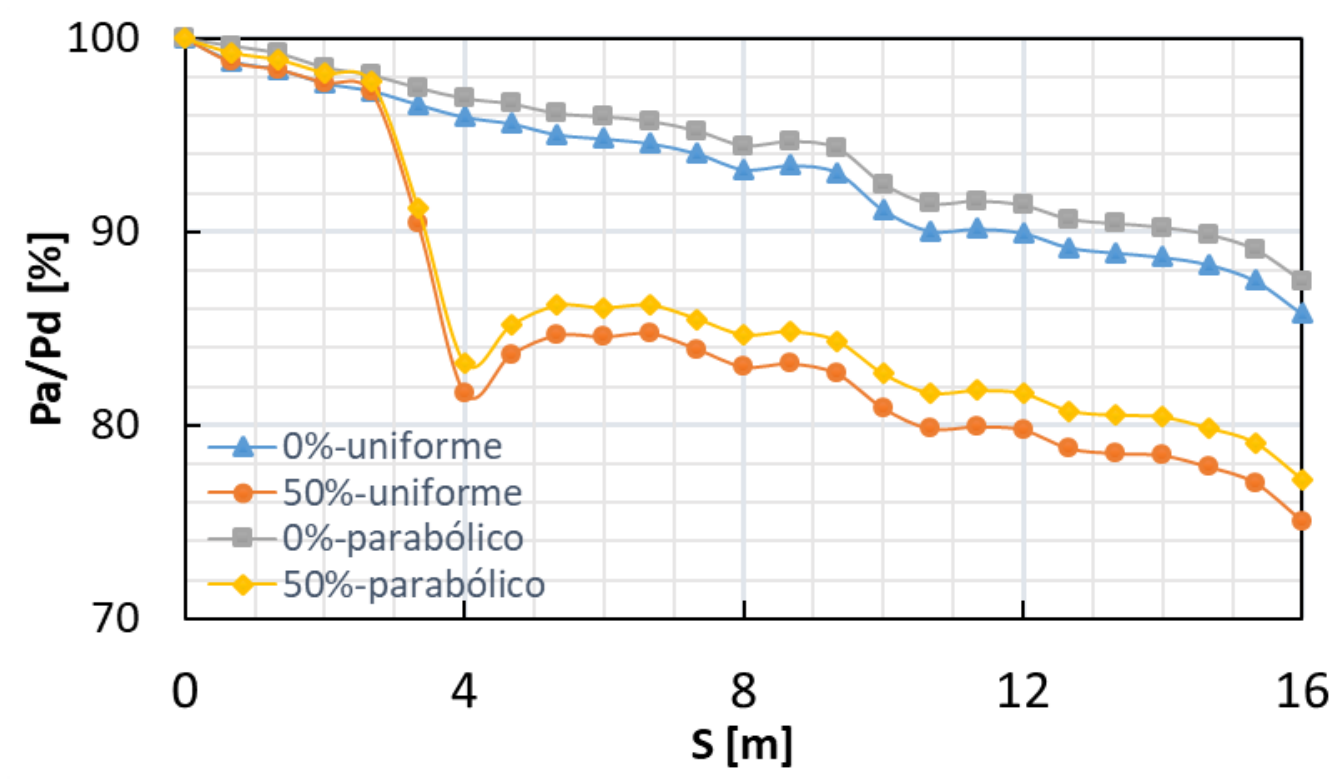

Figura 4.3 - Influência do perfil de velocidade na entrada da distribuição de pressão

A geometria utilizada para este gráfico foi substituída por uma similar, para a realização dos cálculos vistos no capítulo 5 de resultados, porém o resultado deve permanecer análogo. 


\subsection{Teste de malha}

Um teste de malha tem sua importância em verificar se a malha a ser utilizada não apresenta grandes diferenças em resultado de uma malha muito mais refinada, ou seja, minimizar o tempo de computação sem perder qualidade da malha. Todas as malhas foram feitas de modo semelhante. O teste de malha consistiu em verificar o resultado principal do trabalho, isto é, como as malhas refletiam a resposta ao FFR.

Nos testes de malha foi utilizado o perfil parabólico para a realização da simulação.

\subsubsection{Teste de malha - coronária saudável}

Como primeiro teste de malha, selecionou-se o caso da coronária saudável ( $0 \%$ de redução de área).

A Figura 4.4 representa a malha mais grosseira, mostrando a vista da entrada em primeiro plano e a área da doença.

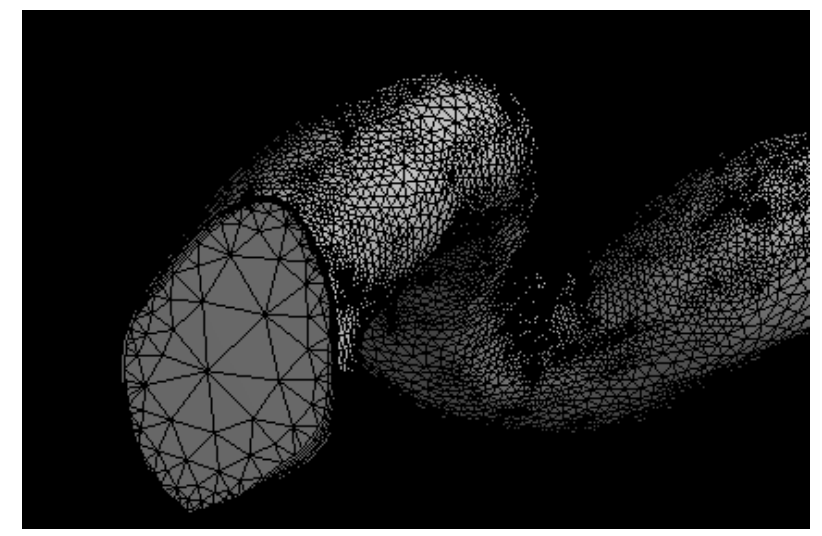

Figura 4.4 - Malha grosseira da coronária saudável

Para este teste quatro malhas distintas foram utilizadas, o número de nós de cada malha está na Tabela 4.1 - Número de nós das malhas. Teste para coronária saudávelTabela 4.1.

Tabela 4.1 - Número de nós das malhas. Teste para coronária saudável

\begin{tabular}{|c|c|}
\hline Malha & Número de Nós \\
\hline Malha 1 & 120.000 \\
\hline Malha 2 & 250.000 \\
\hline
\end{tabular}




\begin{tabular}{|l|l|}
\hline Malha 3 & 410.000 \\
\hline Malha 4 & 760.000 \\
\hline
\end{tabular}

A

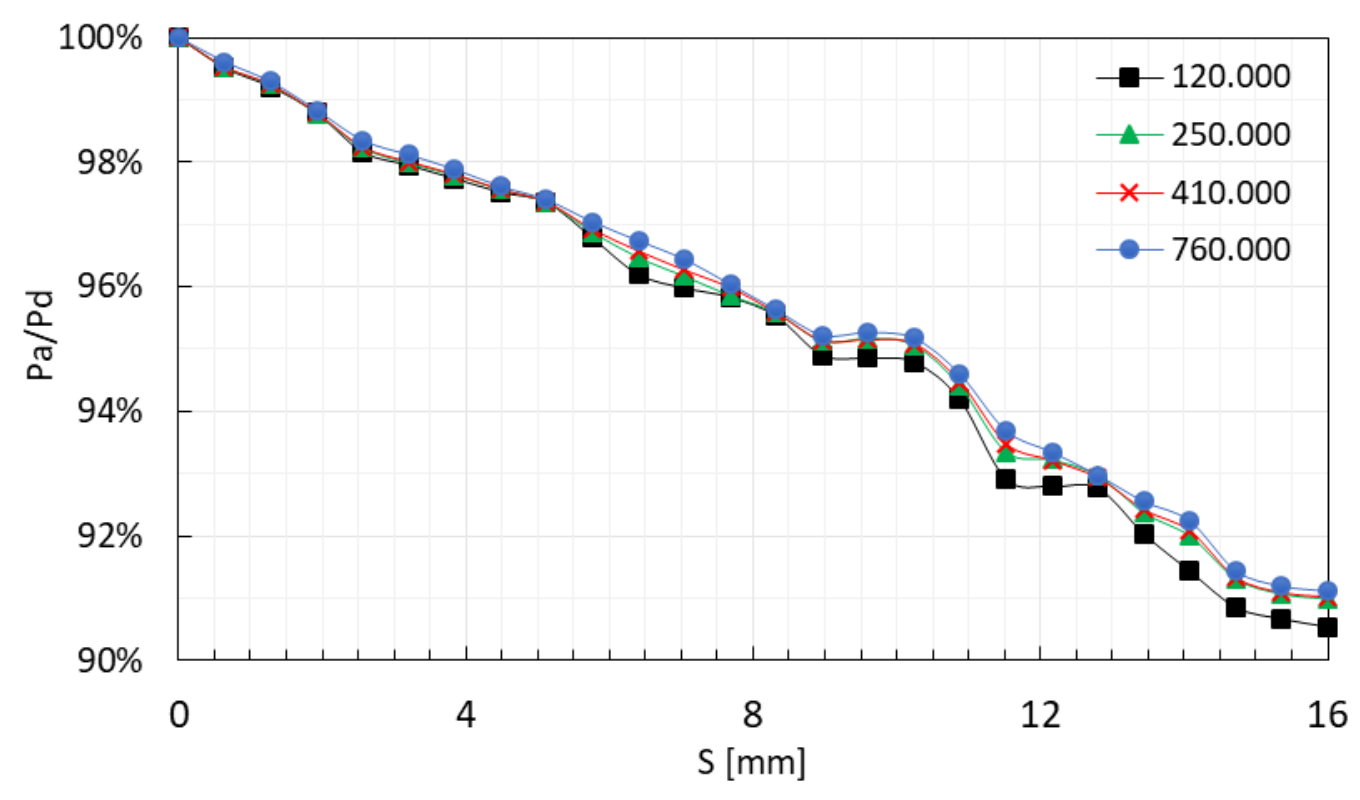

Figura 4.5 - Gráfico da queda de pressão

Erro! Fonte de referência não encontrada. apresenta a variação da pressão ao longo dos planos de referência criado, ilustrada na Figura 4.2, de forma a ilustrar a medida FFR. A curva correspondente a malha mais grossa, 120 mil nós, diverge das outras curvas após os $8 \mathrm{~mm}$, o que a torna inviável. As curvas intermediárias têm resultados bem próximos a mais refinada, de 760 mil nós. A malha selecionada foi a de 410 mil nós, pois mostrou-se que os resultados são suficientemente próximos dos resultados da malha mais refinada. 


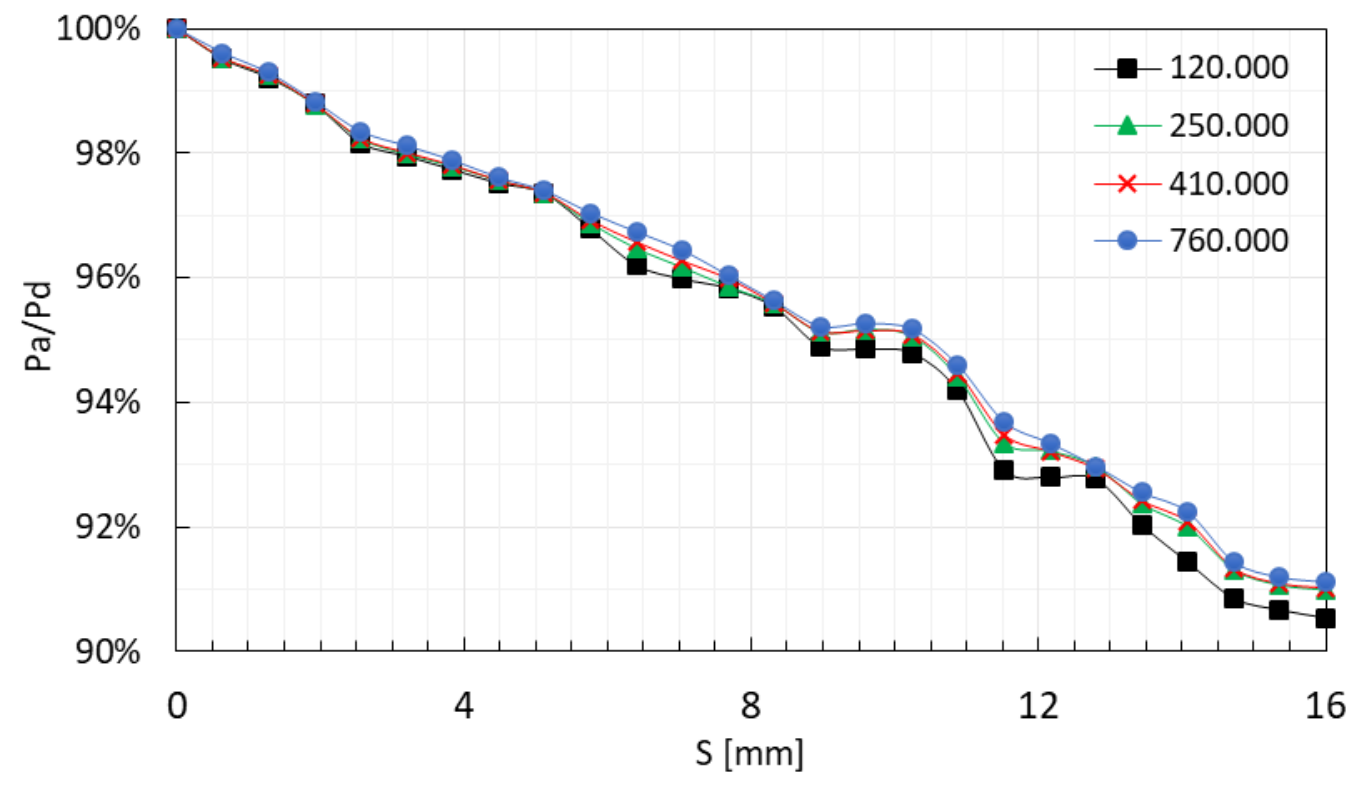

Figura 4.5 - Gráfico da queda de pressão

\subsubsection{Teste de malha - coronária doente}

Para definir a malha para a coronária doente, selecionou-se o caso com $70 \%$ de redução de área. De forma análoga ao utilizado no teste anterior, avaliou-se a variação da pressão ao longo da coronária, informação necessária para o teste FFR. A Erro! Fonte de referência não encontrada.Figura 4.6 representa a malha mais grosseira, visualizando a entrada em primeiro plano e a área da doença na parte posterior.

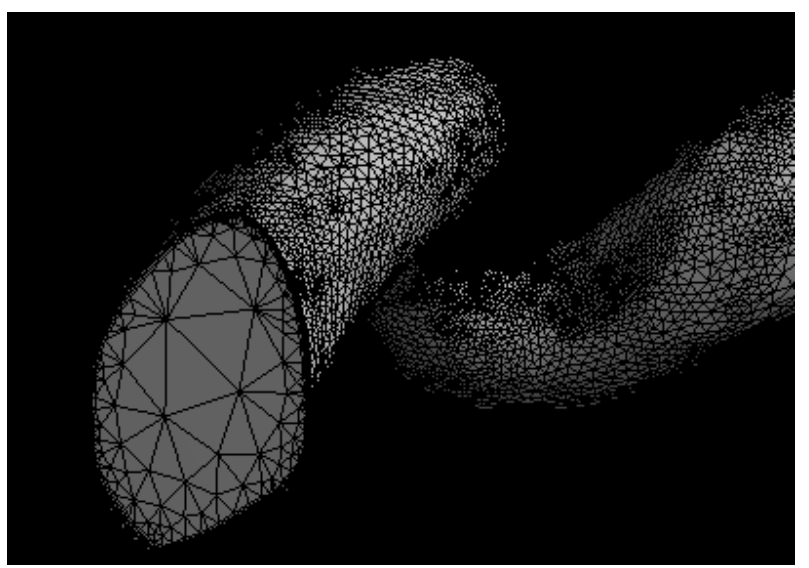

Figura 4.6 - Malha grosseira da coronária doente $70 \%$ 
Para este teste de malhas também foram utilizadas quatro malhas distintas, o número de nós de cada malha está na Tabela 4.2.

Tabela 4.2 -Tabela de número de nós das malhas

\begin{tabular}{|c|c|}
\hline Malha & Número de Nós \\
\hline Malha 1 & 120.000 \\
\hline Malha 2 & 210.000 \\
\hline Malha 3 & 390.000 \\
\hline Malha 4 & 760.000 \\
\hline
\end{tabular}

A

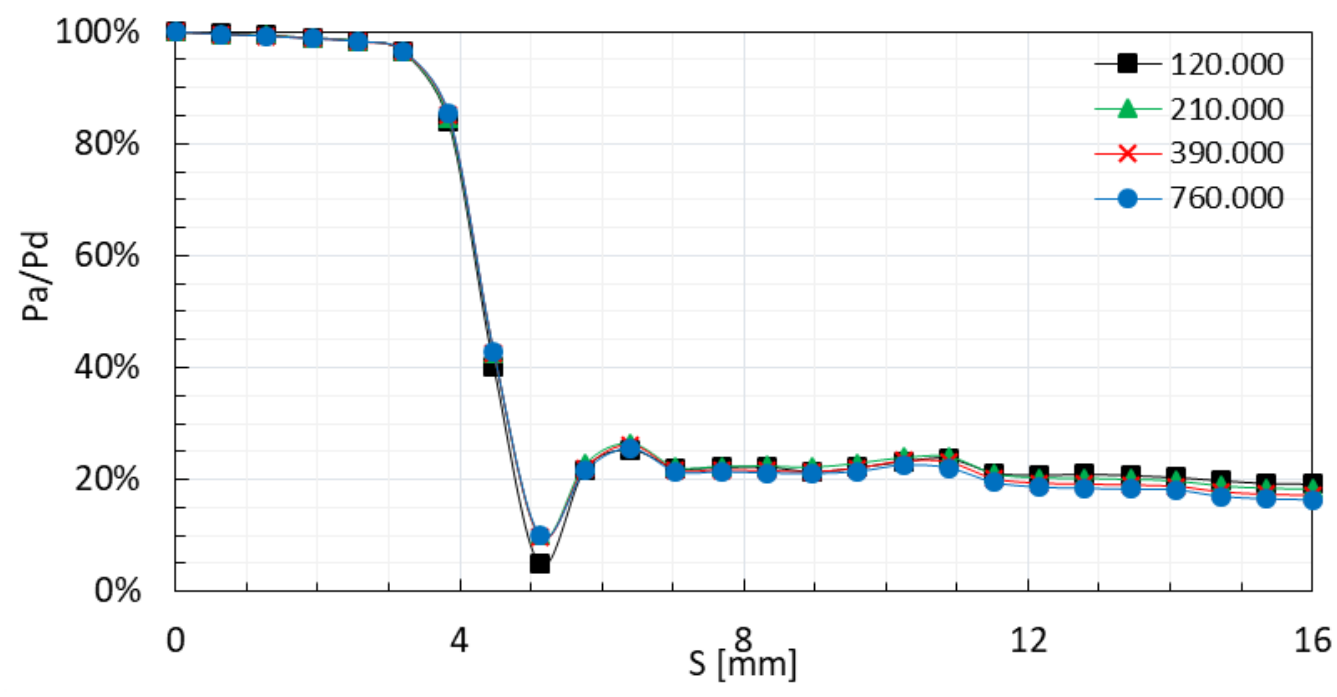

Figura 4.7 apresenta o perfil de pressão ao longo da coronária correspondente a este teste de malha. A malha mais grossa apresentou um resultado muito pior que as outras a tornando inviável. As malhas intermediárias apresentam resultados similares, porém o perfil de pressão da malha de 210 mil nós diverge dos demais dados, próximo ao fim da seção da coronária. 


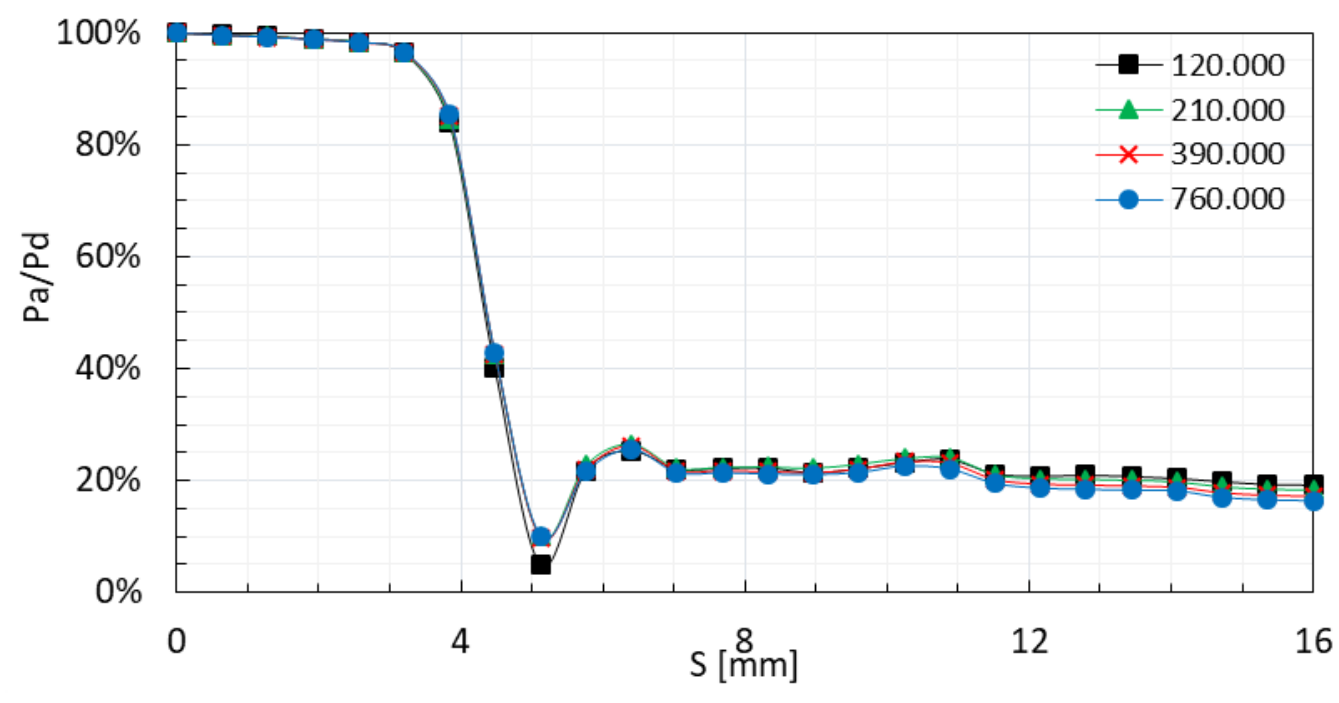

Figura 4.7 -Gráfico da queda de pressão

A malha utilizada foi a de 390 mil nós, que obteve resultados, principalmente na área de interesse, muito próximos aos da malha mais refinada 760 mil nós. 


\section{5 \\ Resultados}

Neste capítulo apresenta-se o efeito de diferentes percentuais de obstrução da coronária no escoamento, em especial na queda de pressão causada pela obstrução.

O sangue foi considerado com fluido Newtoniano com propriedades constantes, as quais foram definidas de acordo com recomendação de Feijó (2007), sendo a massa específica definida como $\rho=1054 \mathrm{~kg} / \mathrm{m}^{3}$ e a viscosidade molecular como $\mu=0,0072 \mathrm{~Pa}$ s.

Considerou-se uma velocidade média na entrada de $0,21 \mathrm{~m} / \mathrm{s}$ o que corresponde a uma vazão típica de $0,000720 \mathrm{~m}^{3} / \mathrm{s}$, sendo a pressão de referência igual a $110 \mathrm{mmHg}$ que corresponde a $14665 \mathrm{~Pa}$.

A seção da coronária obtida do exame de um paciente, possui área de entrada aproximadamente igual a uma elipse com eixos principais iguais a $r_{a}=3,95 \mathrm{~cm}$ e $r_{b}=2,76 \mathrm{~cm}$, resultando num diâmetro hidráulico igual a $6,45 \mathrm{~cm}$.

Os dados apresentados nesta seção correspondem a um número de Reynolds igual a $\mathrm{Re}=1982$.

A partir do teste de malha apresentado no capítulo anterior, selecionou-se uma malha com aproximadamente 400 mil nós para todos os casos.

Definiu-se arbitrariamente dois planos $+X$ e $-X$ para representar duas vistas distintas da coronária. Sendo $+X$ o plano em que o eixo $x$ aponta para o observador $\mathrm{e}-\mathrm{X}$ o plano em que o eixo $\mathrm{x}$ aponta para o lado oposto ao observador.

Para a visualização do campo de velocidade ao longo da coronária foram criados diversos planos transversais a coronária. A Figura 5.1 ilustra o comportamento da velocidade, através de vetores. Como esperado, conforme o diâmetro diminui a velocidade aumenta na área da 
estenose. Uma seta foi inserida na figura para ajudar na interpretação da direção do fluido, assim como a localização da entrada e da saída da coronária.

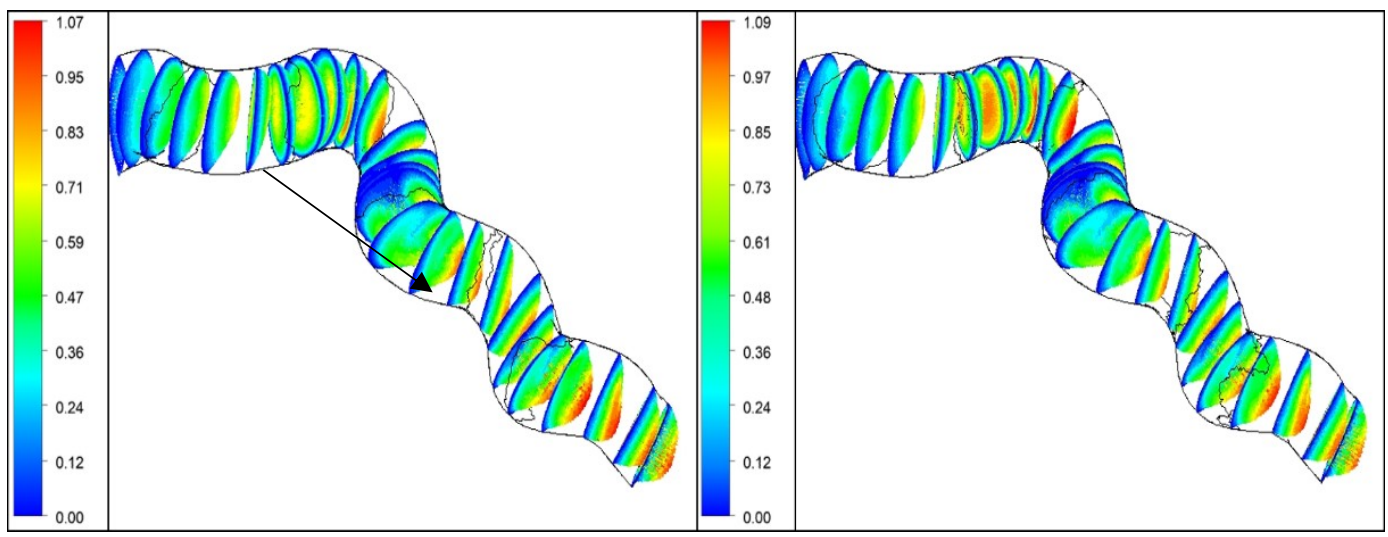

(a) a esquerda $0 \%$ e a direita $16 \%$

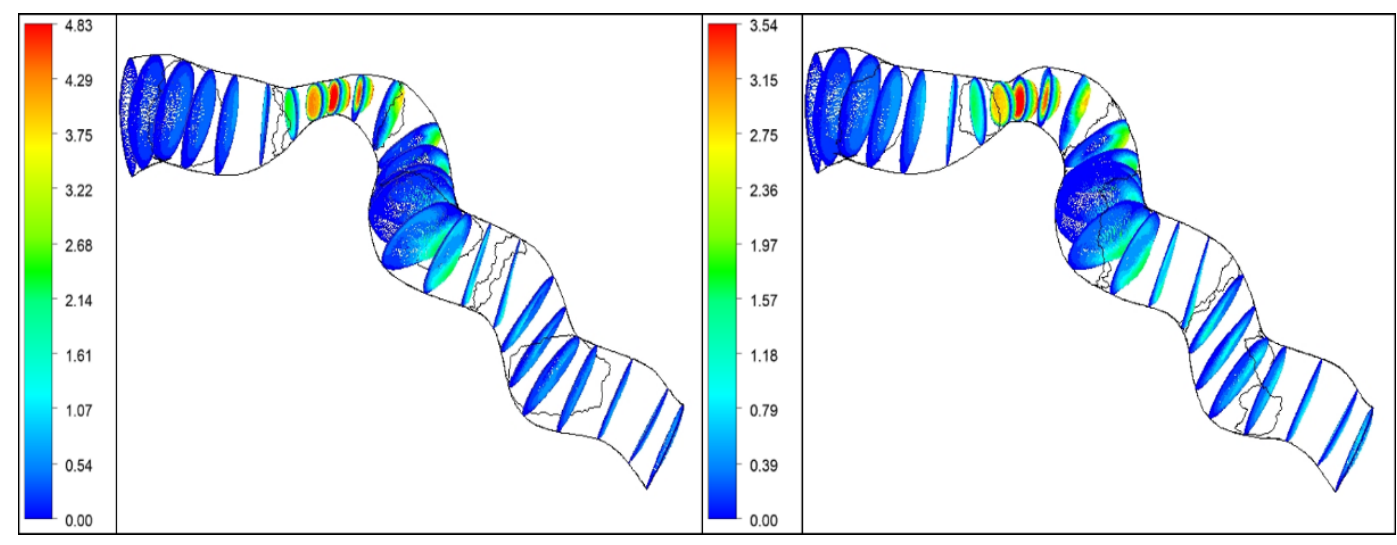

(b) a esquerda $34 \%$ e a direita $50 \%$

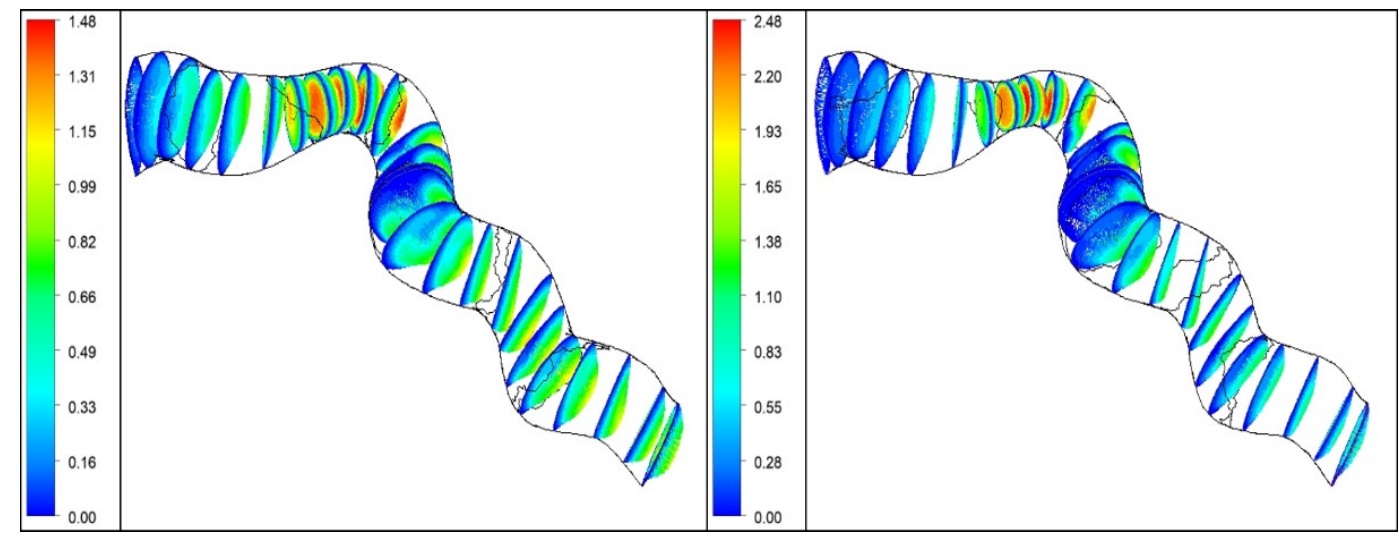

(c) a esquerda $60 \%$ e a direita $70 \%$

Figura 5.1 - Planos de velocidade no plano +X, para a coronária saudável e com as diferentes obstruções 
A Figura 5.2 e a Figura 5.3 apresentam a distribuição de tensão cisalhantes nas paredes da coronária. A Figura 5.2 apresenta a coronária saudável, e as com $16 \%$ e $34 \%$ de redução de área, enquanto a Figura 5.3 apresenta as coronárias com 50\%,60\% e 70\% de redução de área. Devido aos diferentes níveis de intensidade de tensão cisalhante dependendo do grau de obstrução, utilizou-se escalas diferentes nas figuras para melhor visualização dos resultados. Obstruções inferiores a $50 \%$ apresentam tensões cisalhantes máximas inferiores a metade do valor máximo observado nos casos de grandes obstruções.

A região afetada pela tensão cisalhante é altamente influenciada pelo nível da estenose, como podemos ver na Figura 5.2 e na Figura 5.3. Também vemos que conforme o diâmetro diminui a tensão cisalhante na parede aumenta significativamente. A diminuição também gera uma tensão maior na parte posterior à região de localização da doença, o que acaba gerando um desgaste maior no tecido da parede coronariana, isso é explicado pela aceleração do fluido ao passar pelo estreitamento.

O campo de pressão nas paredes da coronária para todos os casos é ilustrado na Figura 5.4 e na Figura 5.5, correspondentes à visão pelo lado $+\mathrm{X}$ ou $-\mathrm{X}$. O mesmo intervalo de pressões foi utilizado para todos os casos.

As pressões, conforme esperado, decaem lentamente devido ao atrito entre o fluido e as paredes, porém podemos notar que, novamente, para pequenas obstruções, inferior a $50 \%$, o decaimento não é tão brusco, porém é possível notar que a queda de pressão aumenta com o incremento da lesão estenótica. O decaimento na obstrução de $70 \%$ vai de $110 \mathrm{mmHg}$ a $20 \mathrm{mmHg}$, correspondendo a um FFR próximo a $20 \%$, o que representa uma doença muito grave. A Figura 5.6 mostra o perfil de queda de pressão em relação à severidade da estenose.

As setas presentes nas figuras Figura 5.2, Figura 5.3, Figura 5.4 e Figura 5.5 existem apenas para facilitar a interpretação, demonstrando a direção do fluxo sanguíneo. 


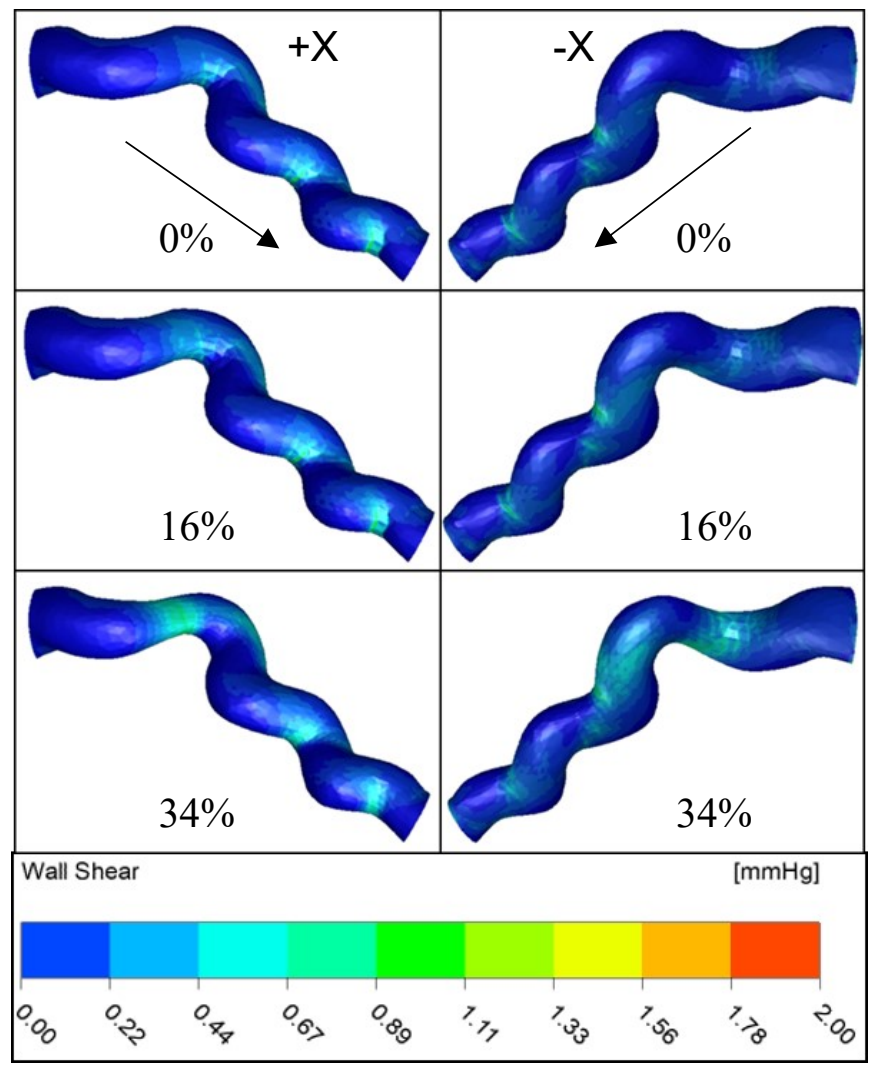

Figura 5.2 - Tensão cisalhante nas coronárias (0\%, 16\%, 34\%).

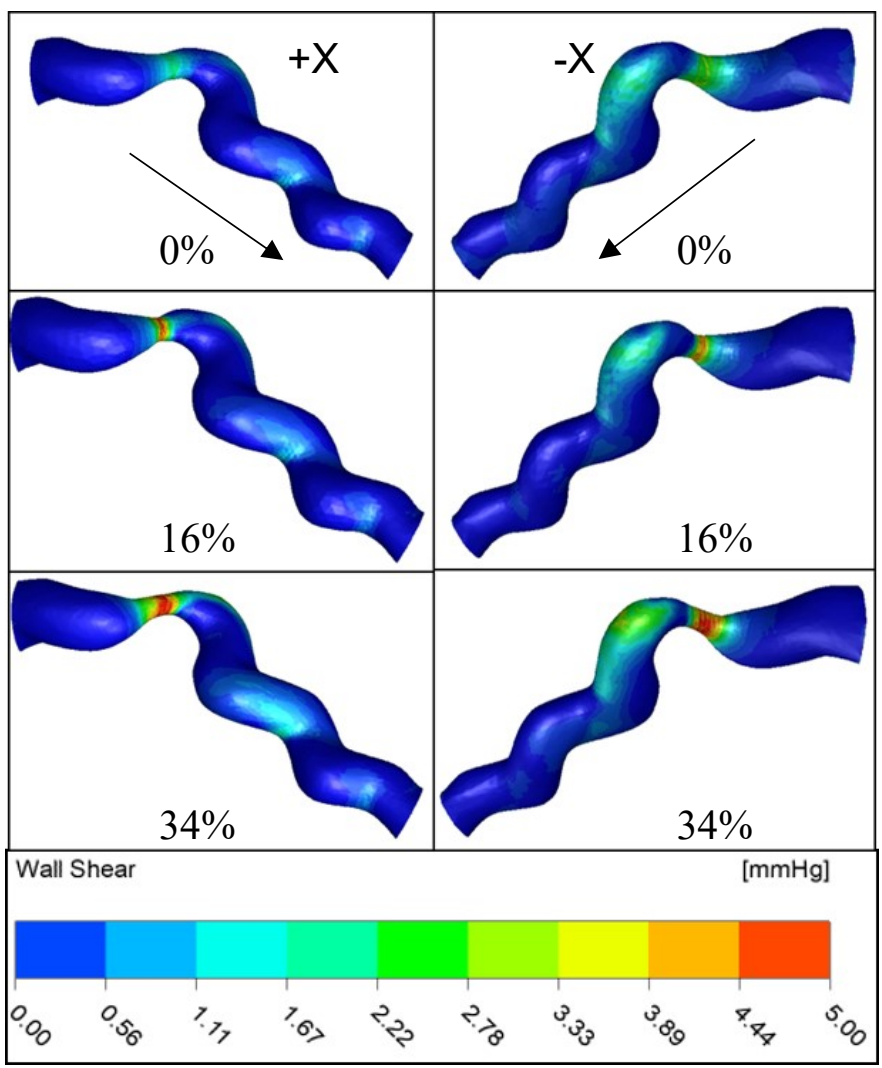

Figura 5.3 - Tensão cisalhante na coronária $(50 \%, 60 \%, 70 \%)$ 


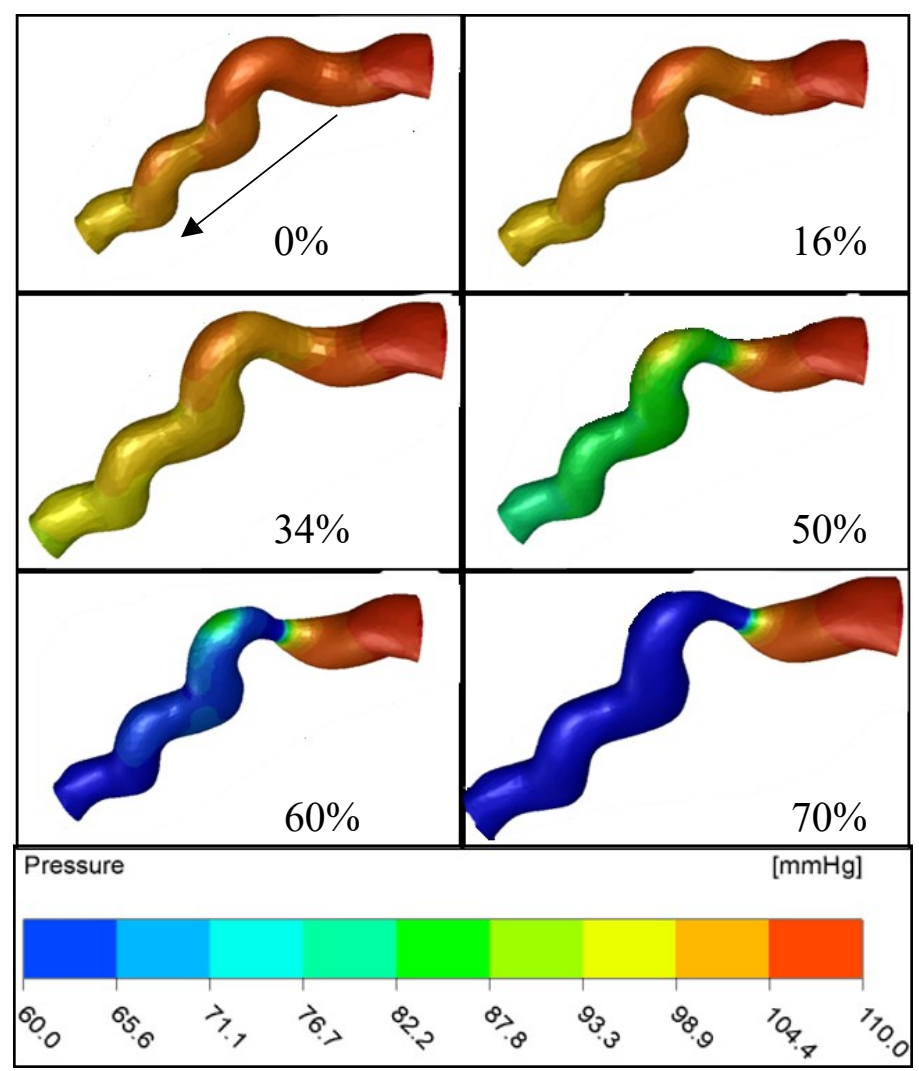

Figura 5.4 - Pressão na parede no plano -X

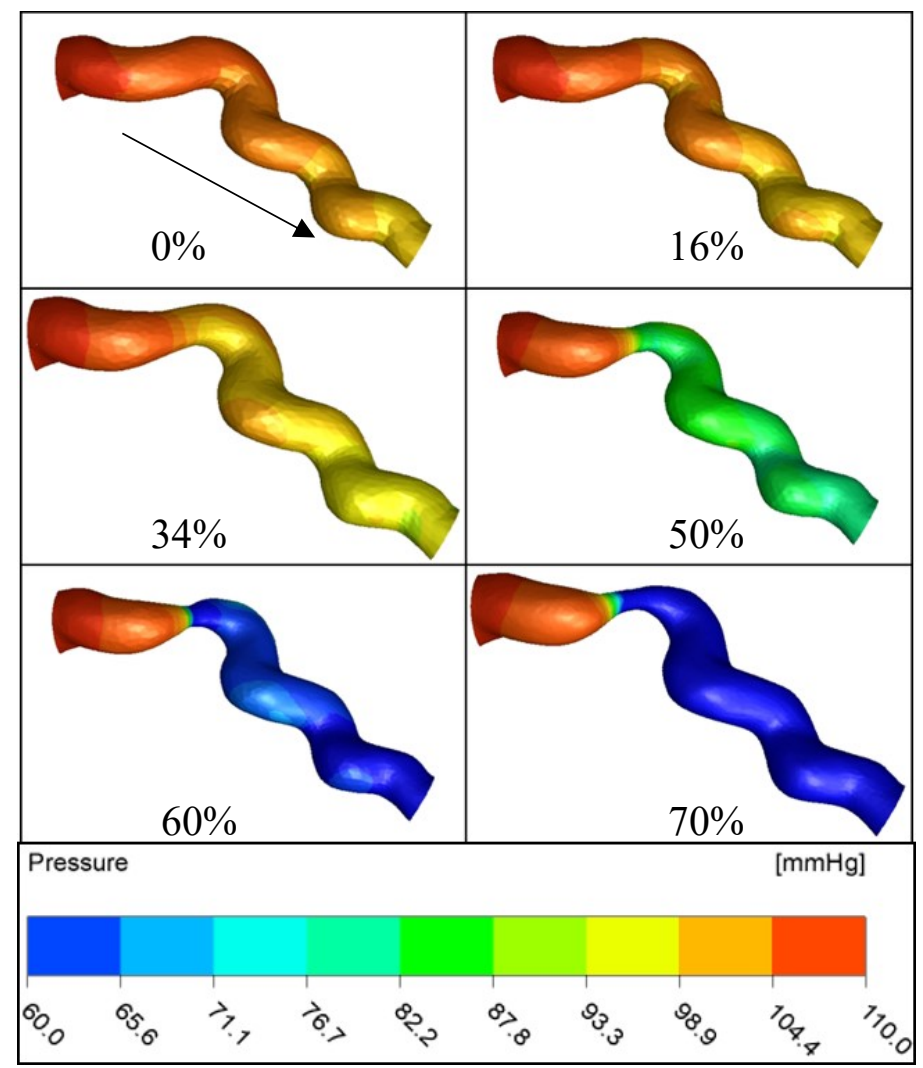

Figura 5.5 - Pressão na parede no plano $+X$ 
A pressão é um resultado muito importante, pois é a partir dela que o FFR é calculado. A FFR é definida como a razão entre a pressão de montante $p_{a}$ e jusante $p_{d}$ da obstrução. A figura 5.6 apresenta na ordenada a FFR e na abscissa a coordenada axial ao longo da coronária. Cada ponto do gráfico representa a média da pressão de um plano, sendo estes planos os mesmos apresentados na Figura 4.2figura 5.1.

Podemos ver que a queda de pressão na passagem pela doença é significativa a partir de $50 \%$, a queda já apresenta uma decaída de aproximadamente $25 \%$ da pressão original o que já é considerado isquemia do miocárdio segundo (Nico H.J. Pijls, et al., 2000) e já é recomendado o tratamento para o paciente.

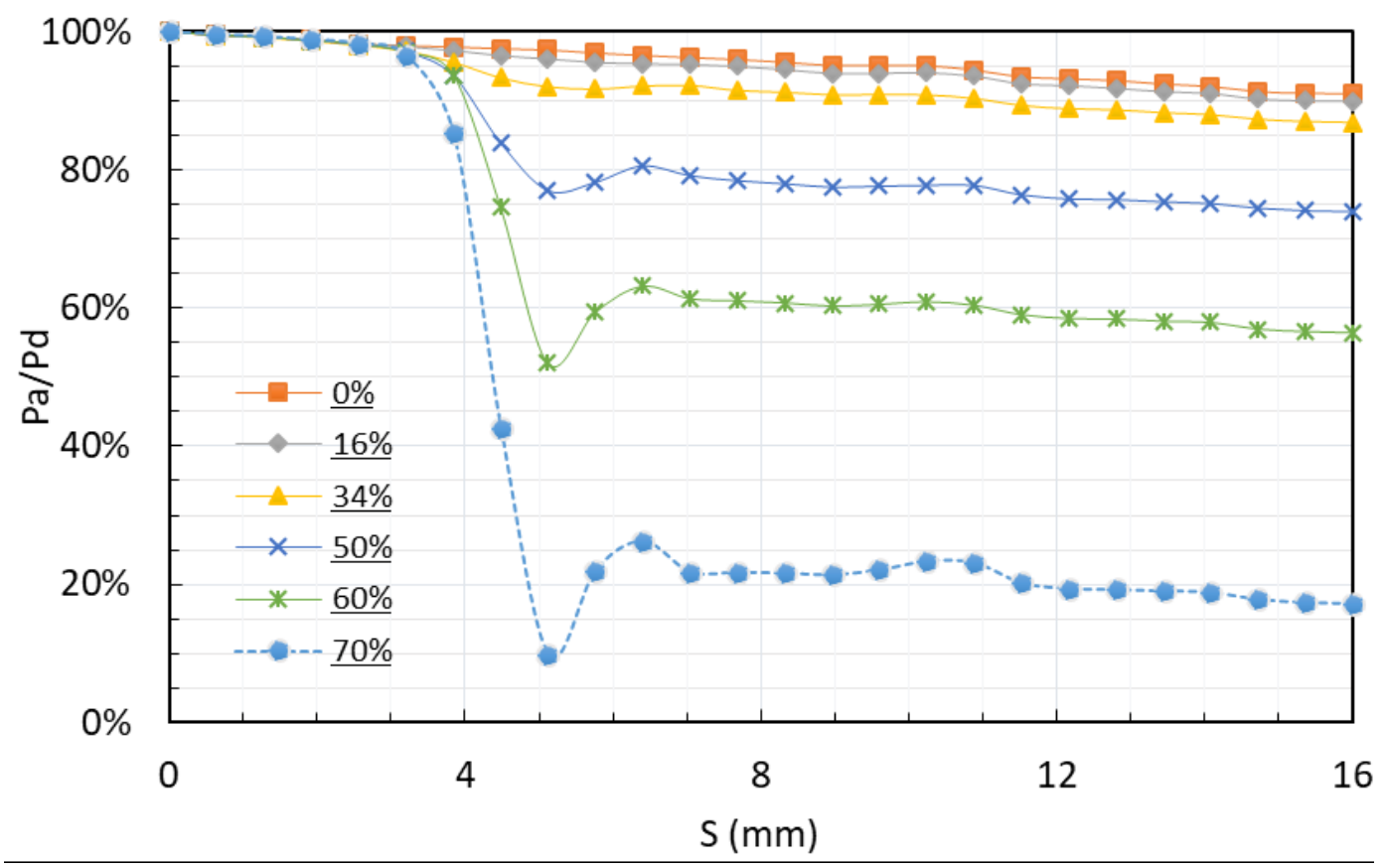

Figura 5.6 - A relação entre $P d / P a$ e a direção axial S.

Na figura 5.7 a abscissa representa a porcentagem de redução de área em relação a área original da artéria coronária, ou seja, os níveis de estenose mencionados anteriormente. Em um caso real, as perdas de carga por atrito no endotélio saudável não são consideradas. Porém, neste estudo, a perda de carga devido ao atrito é levada em consideração, ocasionando, mesmo na coronária saudável, um FFR abaixo de 100\%. A Figura 5.7 apresenta um gráfico normalizado pela 
coronária saudável para uma visão mais ideal, a queda de pressão no caso foi de aproximadamente $9 \%$. A linha do FFR é representada por uma linha sólida preta, enquanto a linha pontilhada em azul é uma linha de tendência. O FFR foi calculado pela razão da pressão da entrada e a pressão média na saída.

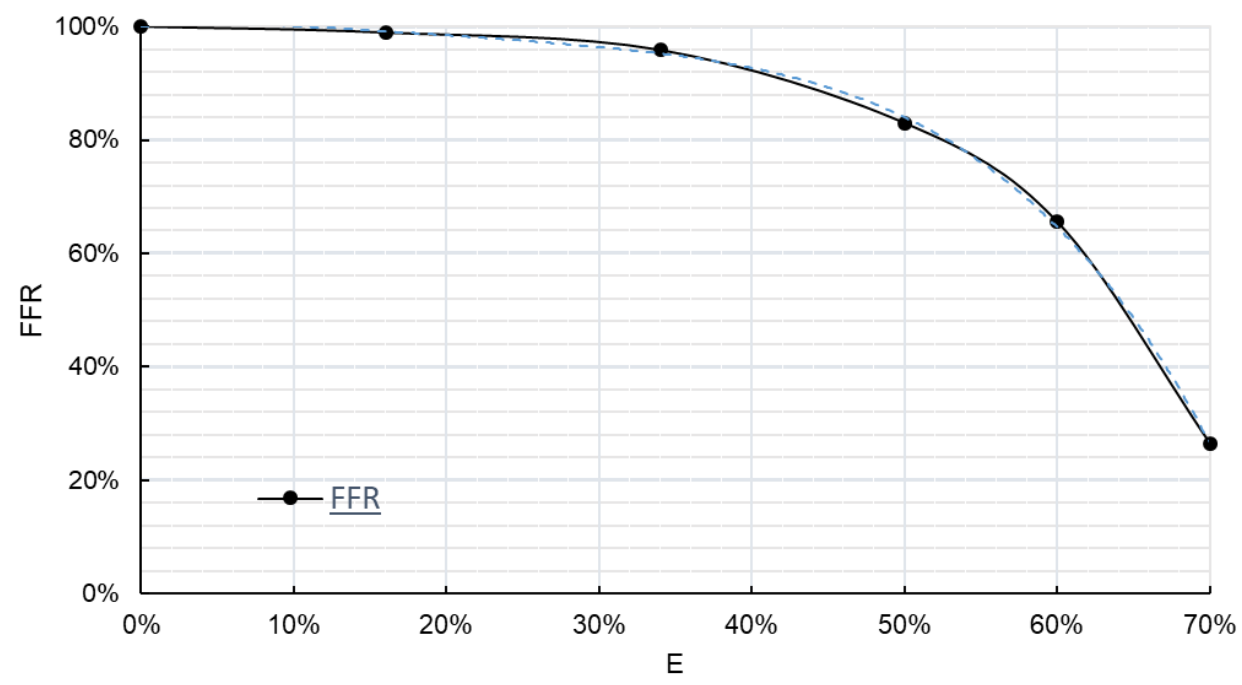

Figura 5.7 - Gráfico do perfil de queda de pressão e sua linha de tendência

A parir dos dados apresentados na figura 5.7 foi possível obter uma linha de tendência da FFR em função da razão de obstrução, a qual é fundamental para auxiliar os médicos a identificarem a necessidade de uma intervenção cirúrgica no paciente.

A linha de tendência, representada pela linha pontilhada na figura 5.7 é a curva que representa a equação

$$
F F R=-9,5839 \alpha^{4}+7,7395 \alpha^{3}-2,4874 \alpha^{2}+0,1875 \alpha+1
$$

sendo a razão de obstrução dada por

$$
\alpha=\frac{A_{\text {obst }}}{A_{\text {saudável }}}
$$




\section{6 \\ Comentários Finais}

No presente trabalho, investigou-se numericamente o escoamento em um trecho de coronária saudável e com diversos níveis de obstrução, determinando a variável FFR, crítica para auxiliar os médicos sobre a necessidade de intervenção cirúrgica em um paciente.

O diagnóstico de um FFR abaixo de $80 \%$ já é considerado uma estenose coronariana significativa. Esta queda de pressão geralmente está associada a estenoses de $50 \%$, o que condiz com os resultados obtidos.

A partir dos resultados obtidos neste trabalho, desenvolveu-se uma correlação (um polinômio de quarta ordem) para determinar o valor da FFR em função da razão de obstrução $\alpha$, isto é, a razão entre a área obstruída causada pela estenose em relação a área saudável.

Como recomendação para trabalhos futuros, recomenda-se investigar a influência da localização da estenose no trecho da coronária selecionado. Recomenda-se ainda avaliar um ramo das artérias coronárias, ou até mesmo, com toda rede arterial, incluindo fluxo pulsátil e parede vascular complacente. Finalmente, recomenda-se ainda representar o sangue de forma mais realista, empregando um modelo reológico mais representativo que o de fluido newtoniano. 


\section{Referências}

Charles A. Taylor, PHD, Timothy A. Fonte, BS e James K. Min, MDz. 2013. Computational Fluid Dynamics Applied to Cardiac Computed Tomography for Noninvasive Quantification of Fractional Flow Reserve. JACC. 04 de 06 de 2013, Vol. 61, 22, pp. 2233-2241.

Eslami, Parastou, et al. 2015. Computational Study of Computed Tomography Contrast Gradients in Models of Stenosed Coronary Arteries. Journal of Biomechanical Engineering. 09 de 2015, Vol. 137, doi: 10.1115/1.4030891.

Feijó, V. 2007. Modelagem do Fluxo Sanguíneo na Aorta Abdominal Utilizando a Interação Fluido-Estrutura. Faculdade de Engenharia de Ilha Solteira, Universidade Estadual Paulista. São Paulo: s.n., 2007. Dissertação (mestrado).

Fox, McDonalds e Pritchard, Philip J. 2011. Introduction to Fluid Mechanics. Manhattan: John Wiley \& Sons, Inc, 2011. Vol. 8. ISBN-13 9780470547557 ISBN-10 0470547553.

Hajati, Omid, et al. 2012. CFD Simulation of Hemodynamics in Coronary Arteries Using Geometry Reconstruction Based on CT Scan Data. International Conference on Mechanical Engineering and Mechatronics. 16-18 de Agosto de 2012, p. 8.

James K. Min, MD, et al. 2012. Diagnostic Accuracy of Fractional Flow Reserve From Anatomic CT Angiography. Jama. [Online] 26 de 07 de 2012. [Citado em: 12 de junho de 2017.] www.jama.com. 10.1001/2012.jama.11274.

Kern, Morton J. e Samady, Habib. 2010. Current Concepts of Integrated Coronary Physiology in the Catheterization Laboratory. JACC. 19 de 01 de 2010, Vol. 55, 3, pp. 173-185.

Nico H.J. Pijls, MD, PhD, et al. 2000. Coronary Pressure Measurement to Assess the Hemodynamic Significance of Serial Stenoses Within One Coronary 
Artery. Circulation. [Online] 07 de 09 de 2000. [Citado em: 12 de junho de 2017.] http://circ.ahajournals.org. ISSN 1524-4539.

Nørgaard, Bjarne L., et al. 2015. Influence of Coronary Calcification on the Diagnostic Performance of CT Angiography Derived FFR in Coronary Artery Disease. JACC: Cardiovascular Imaging. 09 de 2015.

NXT Trial. 2014. Diagnostic Performance of Noninvasive Fractional Flow Reserve Derived From Coronary Computed Tomography Angiography in Suspected Coronary Artery Disease. JACC. 2014, Vol. 63, 12.

Rohsenow, Warren M., Harnett, James P. e Cho, Young I. 1998. Handbook of Heat Transfer. s.1. : McGraw Hill, 1998. ISBN 0-07-053555-8 . 